\title{
On Effective Young's Modulus and Poisson's Ratio of the Auxetic Thermoelastic Material
}

\author{
B.T. Maruszewski, A. Drzewiecki, R. Starosta \\ Poznan University of Technology, Institute of Applied Mechanics \\ Jana Pawta II 24, 60-965 Poznan \\ E-mail: bogdan.maruszewski@put.poznan.pl,andrzej.drzewiecki@put.poznan.pl,roman.starosta@put.poznan.pl
}

Received: 11 November 2016; revised: 06 December 2016; accepted: 07 December 2016; published online: 30 December 2016

\begin{abstract}
The paper deals with an influence of the excitation frequency and the dimensions of a free supported thermoelastic plate on the effective Poisson's ratio and the effective Young's modulus. Both of these parameters are not, in such a situation, the elastic material constants. The considered thermoelastic problem has been modelled within the extended thermodynamical model. Therefore, the above effective elastic coefficients are also dependent on the thermal relaxation time. The numerical analysis of those coefficients vs. excitation frequency both for normal and auxetic plates have been presented.
\end{abstract}

Key words: effective elastic coefficients, auxetics, thermoelastic damping

\section{INTRODUCTION}

Thermoelastic problems have been considered for years by numerous authors (cf. [1-4]).

Most of them refer to a situation when the elastic parameters as constants have been used. However, during dynamical thermoelastic interactions, particularly in bodies of finite extent, the elastic parameters, i.e. Young's modulus and Poisson's ratio, depend on frequency, thermal relaxation time and dimensions of the body [5-7]. In such dynamical situations also a peculiar phenomenon called the thermoelastic damping occurs.

In the case of a plate, for instance, there occurs an additional energy dissipation coming from an additional heat flux normal to the middle surface during its vibrations. The origin of that flux is the alternate compression and extension of upper and lower fibers of that body. This way, in the case of the plate the problem is $2 \mathrm{D}$ (plate) - 3D (additional dimension resulting from its thickness). [8] was the first to point out that the thermoelastic damping comes from heterogeneities giving rise to fluctuations of temperature. That idea was developed by [7,9] and then by [10]. Since the investigated elastic parameters also depend on the thermal relaxation time, to be more close to reality [11] have proposed a description of thermoelastic damping within the extended thermodynamical model (cf. [12-14]) which is crucial in nanoscience and engineering. That model deals with the description proving that the thermal signals propagate with finite velocity. Simultaneously, taking into account the dynamical character of thermoelastic interactions with the thermoelastic damping in a body of finite extent within the extended thermodynamical model a detailed discussion of the properties of the elastic parameters becomes possible.

Since in contemporary technologies one applies a very broad range of materials of sometimes very peculiar properties, the above discussion is carried out in the paper both for normal and auxetic materials (materials of negative Poisson's ratio) [15-21] proved that Poisson's ratio is admissible from the thermodynamical point of view satisfying inequality $-1<\nu<0.5$.

Negative Poisson's ratio materials and structures expand transversely when stretched axially, and also undergo transverse contraction under axial compression. This is shown in Fig. 1, along with the classic example of a re-entrant honeycomb structure deforming by hinging of the cell walls leading to negative Poisson's ratio behavior. There is increasing interest in the development of these novel materials due to their counter-intuitive behavior and also in applications where the 
auxetic property itself, or enhancements in other materials properties due to the negative Poisson's ratio, may be exploited [22].

a)
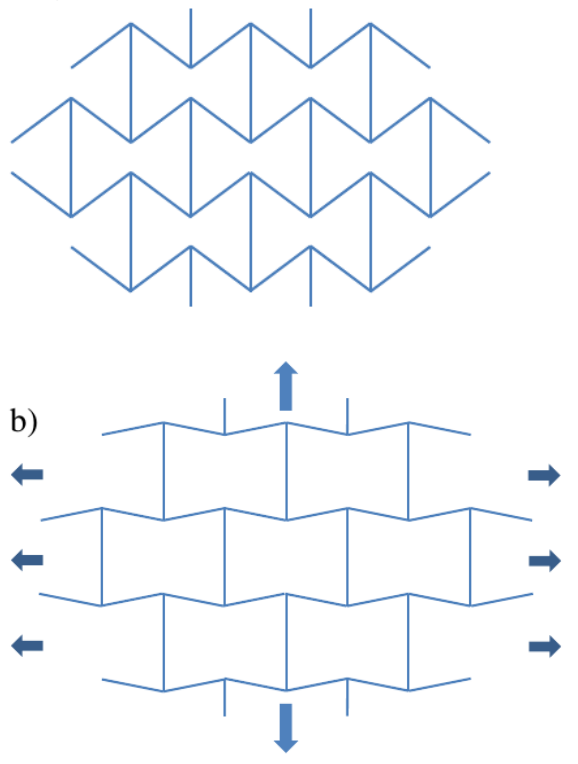

Fig. 1. a) undeformed auxetic; b) deformed auxetic paper. The authors have chosen only fundamental positions which were, in their opinion, sufficient to the suitable description of the problem considered in the paper.

\section{BASIC THEORY}

Since we are interested in determination and analysis of Young's modulus-like and Poisson's ratio-like effective parameters, one of the methods to do that is an investigation of the forced vibrations of a thermoelastic body with the forcing frequency $\omega$.

Therefore, let us consider a simply supported thermoelastic rectangular plate $0 \leq x_{1} \leq a, 0 \leq x_{2} \leq b,-\frac{h}{2} \leq x_{3} \leq \frac{h}{2}$. Following [5,6] the effective parameters $E$ and $\nu$ read for

1 . very low frequency $\omega$ approximation [7]

$$
E=E_{T}=\text { const }, \nu=\nu_{T}=\text { const },
$$

(they have constant values which are the same if they result from the classical and the extended thermodynamical models),

2. very high frequency $\omega$ approximation

$$
E=\hat{E}_{s}=\text { const }, \nu=\hat{\nu}_{s}=\text { const }
$$

$\left(E_{s} \neq \hat{E}_{s}, \nu_{s} \neq \hat{\nu}_{s} ; E_{s}, \nu_{s}\right.$ result from the classical and $\hat{E}_{s}, \hat{\nu}_{s}$ result from the extended thermodynamical models),

3 . for arbitrary frequency $\omega$ approximation

$$
\begin{aligned}
E & =E_{T} \frac{1-\nu^{2}}{1-\nu_{T}^{2}}-\left(E_{s}-E_{T}\right) \\
& \times \frac{1-2 \nu}{1-2 \nu_{s}}\left(1+\nu_{T}\right) \frac{1-\nu^{2}}{1-\nu_{T}^{2}} \frac{\omega^{2}\left[1-\tau_{T}^{2}\left(\omega^{2}-\omega_{x}^{2}\right)\right]}{\tau_{T}^{2}\left(\omega_{x}^{2}-\omega^{2}\right)^{2}+\omega^{2}},
\end{aligned}
$$

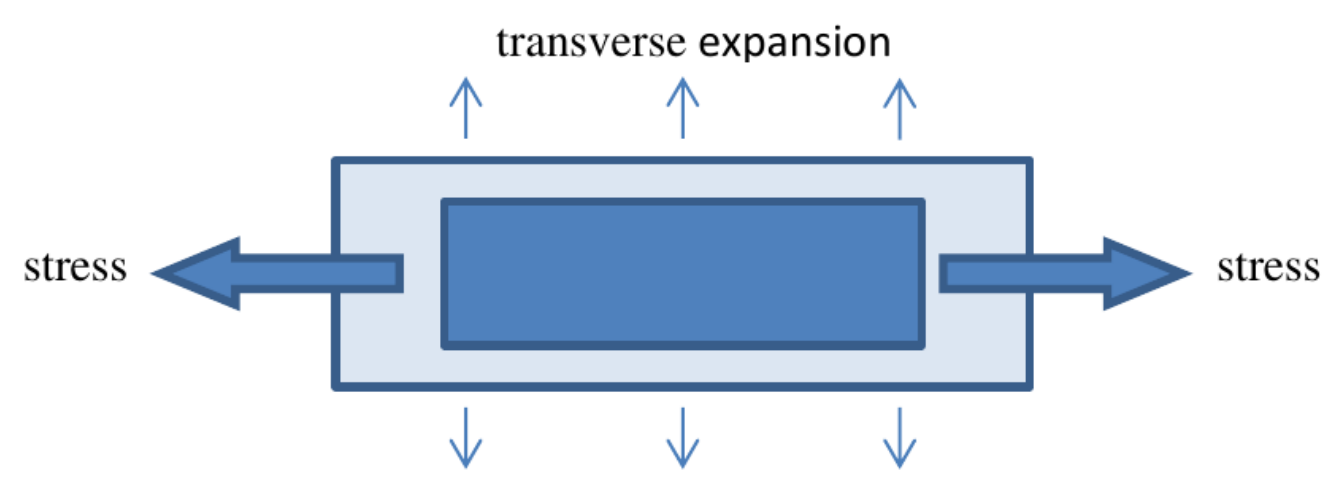

Fig. 2. Continuous macroscopic model of an auxetic 


$$
\begin{aligned}
& \left(1-\nu_{T}\right)\left(1-2 \nu_{s}\right)=(1-\nu)\left(1-2 \nu_{s}\right)-\left(\nu_{s}-\nu_{T}\right) \\
& \times(1-2 \nu)(1-\nu) \frac{\omega^{2}\left[1-\tau_{T}^{2}\left(\omega^{2}-\omega_{x}^{2}\right)\right]}{\tau_{T}^{2}\left(\omega_{x}^{2}-\omega^{2}\right)^{2}+\omega^{2}} .
\end{aligned}
$$

Index " $T$ " denotes the isothermal coefficient and " $s$ " the adiabatic one [1, 7]. The relations (3) and (4) have been derived for the isothermal values [7] of the below coefficients, so

$$
\omega_{x}^{2}=\frac{H k_{T}}{\tau_{T} \rho_{T} c_{v T}}, \quad H=\frac{\pi^{2}}{a^{2}}+\frac{\pi^{2}}{b^{2}}
$$

where $k_{T}$ denotes the heat conduction coefficient, $\rho_{T}$ is the mass density, $c_{v T}$ is the heat capacity in constant volume and $\tau_{T}$ is the thermal relaxation time resulting from the extended thermodynamical model (hyperbolic-type heat conduction equation [5,6]). $\omega_{x} \neq 0$ if the dimensions of the plate $a, b$ are finite. Otherwise, if $a \rightarrow \infty$ and $b \rightarrow \infty$ the problem becomes 2D (two dimensional) and relations in such a case do not hold true because in that situation the definition of Poisson's ratio is different from that concerning 3D situation [16].

\section{NUMERICAL RESULTS}

The introductory analysis of (3) and (4) shows that the effective Young's modulus $E$ and the effective Poisson's ratio $\nu$ depend on the forcing frequencies, i.e. $E=E(\omega)$ and $\nu=\nu(\omega)$. That dependence increases if the frequency $\omega$ increases. Moreover, those relations indicate that the problem is independent of the plate thickness $h$. From (5) it results that for high values of the thermal relaxation time $\tau_{T}$ the influence of the plate dimensions $a, b$ on the investigated processes disappears. The problem becomes 2D. So, although the relations of the effective parameters $E$ and $\nu((3),(4))$ are built on the dimensions of the plate $a, b$, the detailed analysis of that fact shows that their influence on the investigated parameters is negligible.

Most interesting is the dependence of the effective parameters $E$ and $\nu$ on the excitation frequency $\omega$ and the thermal relaxation time $\tau_{T}$.

Let us consider a rectangular normal and auxetic plate of the following geometric and material properties:

$$
\begin{aligned}
& a=1 \mathrm{~m}, \quad b=1 \mathrm{~m}, c_{v T}=460 \mathrm{~J}(\mathrm{~kg} \mathrm{~K})^{-1}, \\
& E_{T}=2 \times 10^{11} \mathrm{~Pa}, E_{s}=2.1 \times 10^{11} \mathrm{~Pa}, \\
& \rho_{T}=7860 \mathrm{~kg} \mathrm{~m}^{-3}, k_{T}=58 \mathrm{~J}\left(\mathrm{~m} \mathrm{~K} \mathrm{~s}^{-1},\right.
\end{aligned}
$$

for the normal plate $\nu_{T}=0.3, \nu_{s}=0.31$, for the auxetic plate $\nu_{T}=-0.3, \nu_{s}=-0.29$.

Young's modulus-like parameters as functions of $\omega$ for normal and auxetic materials are presented in Figs. 3 and 4.
The quantity unit of $E$ is $\mathrm{Pa}$ and the quantity unit of $\omega$ (also in Figs. 5 and 6) is $\mathrm{s}^{-1}$.

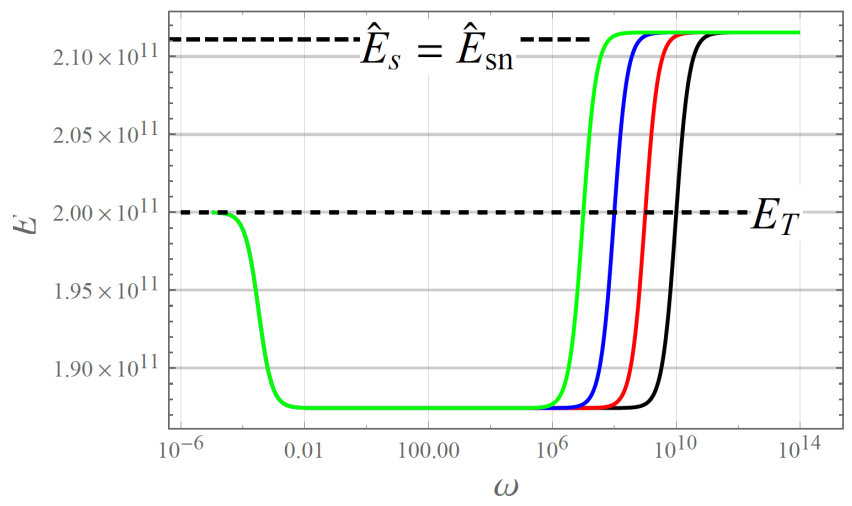

Fig. 3. Effective Young's moduli for normal materials as functions of $\omega$ for different relaxation times $\tau_{T}$ : black line $-\tau_{T}=10^{-10} \mathrm{~s}$, red line $-\tau_{T}=10^{-9} \mathrm{~s}$, blue line $-\tau_{T}=10^{-8} \mathrm{~s}$, green line $-\tau_{T}=10^{-7} \mathrm{~S}$

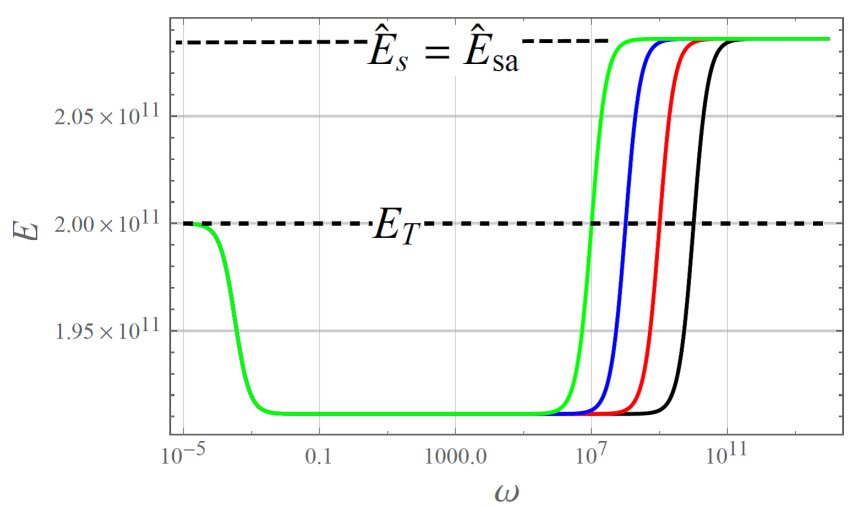

Fig. 4. Effective Young's moduli for auxetic materials as functions of $\omega$ for different relaxation times $\tau_{T}$ : black line $-\tau_{T}=10^{-10} \mathrm{~s}$, red line $-\tau_{T}=10^{-9} \mathrm{~s}$, blue line $-\tau_{T}=10^{-8} \mathrm{~s}$, green line $-\tau_{T}=10^{-7} \mathrm{~S}$

From Figs. 3 and 4 it results that the effective Young's modulus has a dispersive character. That fact confirms the assumption (2) because for very high frequency $\omega$ both for the normal and auxetic material $\hat{E}_{s}=$ const having different values to each other. Fig. 4 shows in comparison to Fig. 3 that the values of the thermal relaxation time $\tau_{T}$ influence the dispersion of the effective Young's modulus much more strongly in the auxetic material than in the classical one. Also the character of that dispersion is different in those materials. Note that during the adiabatic process (for high frequency $\omega)$ the normal material is more rigid than the auxetic one, because

$$
\hat{E}_{s n}>\hat{E}_{s a}, \hat{E}_{s n}=\text { const, } \hat{E}_{s a}=\text { const. }
$$

The influence of $\tau_{T}$ on the $\nu(\omega)$ function for the normal and auxetic materials is presented in Figs. 5 and 6. 


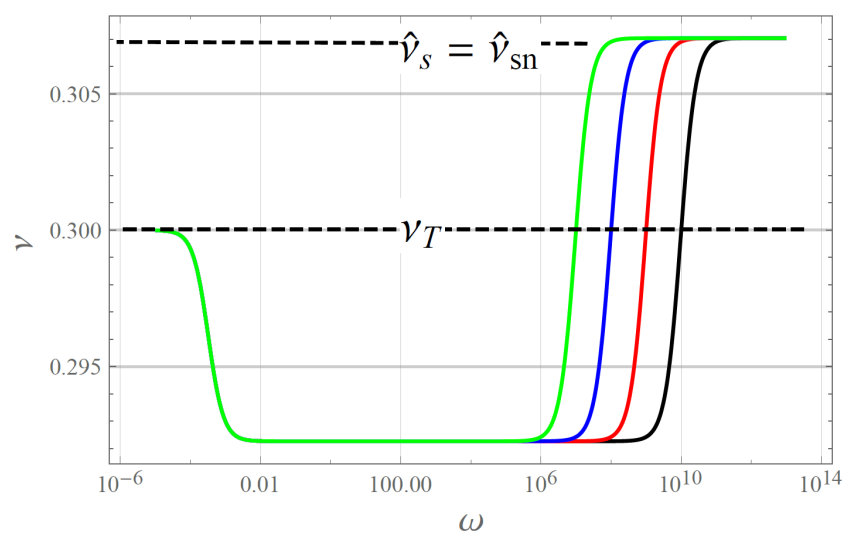

Fig. 5. Effective Poisson's ratio for normal materials as functions of $\omega$ for different relaxation times $\tau_{T}$ : black line $-\tau_{T}=10^{-10} \mathrm{~s}$, red line $-\tau_{T}=10^{-9} \mathrm{~s}$, blue line $-\tau_{T}=10^{-8} \mathrm{~S}$, green line $-\tau_{T}=10^{-7} \mathrm{~S}$

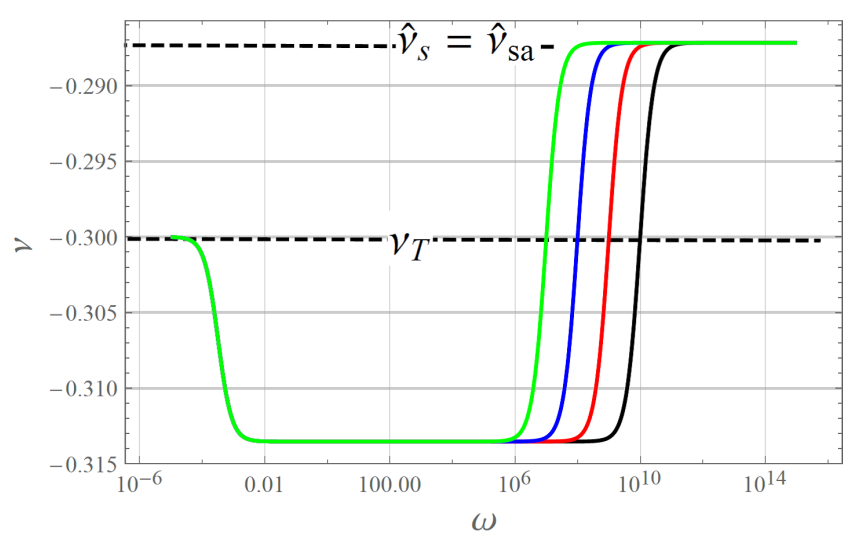

Fig. 6. Effective Poisson's ratio for auxetic materials as functions of $\omega$ for different relaxation times $\tau_{T}$ : black line $-\tau_{T}=10^{-10} \mathrm{~s}$, red line $-\tau_{T}=10^{-9} \mathrm{~s}$, blue line $-\tau_{T}=10^{-8} \mathrm{~s}$, green line $-\tau_{T}=10^{-7} \mathrm{~S}$

Also from Figs. 5 and 6 it results that the effective Poisson's ratio has a dispersive character, too. The characters of those dispersions are similar but the influence of the thermal relaxation time $\tau_{T}$ on that phenomenon is the same. So, contrary to the effective Young's modulus for normal vs. auxetic material (Figs. 3 and 4), the effective Poisson's ratio both for normal and auxetic materials behaves vs. $\omega$ and $\tau_{T}$ in the same way.

\section{CONCLUSIONS}

Discussion presented in the paper proves that the coefficients characterizing a particular material are not always in the form of constants. The considered Young's modulus and Poisson's ratio effective parameters only for elastostatic or thermoelastostatic problems can be taken as constants. In case of dynamical thermoelastic ones those parameters are functions of frequency. Moreover, the significant influence on those coefficients has the thermal relaxation time $\tau_{T}$.

We have also considered materials both of normal and auxetic properties. For the low excitation frequency Young's effective modulus for the normal and auxetic material is the same (see (1), (2) confirmed by (3), (4)). For the high frequencies $\omega$ the normal material is more rigid than the auxetic one. For the low frequencies materials of the definite thermoelastic properties can exist both in the normal and auxetics states. Then for the high frequencies they exist also in both states having, however, different material properties. The effective Young's moduli reached the finite $\hat{E}_{s n}$ and $\hat{E}_{s a}$ values in the asymptotic way, as well as the effective Poisson's ratio $\hat{\nu}_{s n}$ and $\hat{\nu}_{s a}$.

\section{Acknowledgements}

This paper was financially supported by the grant 02/21/DSPB/3477.

\section{References}

[1] W. Nowacki, Thermoelasticity, Pergamon, Oxford 1962.

[2] W. Nowacki, Dynamic problems of thermoelasticity, Noordhoff, Leyden 1975.

[3] B.A. Boley, J.H. Weiner, Theory of thermal stresses, Wiley, New York - London 1960.

[4] N. Noda, R.B. Hetnarski, Y. Tanigawa, Thermal stresses, Taylor \& Francis, New York - London 2003.

[5] B.T. Maruszewski, A. Drzewiecki, R. Starosta, Thermoelastic damping and thermal relaxation time in auxetics, Applied Mechanics and Materials, 432, 215-220 (2013).

[6] B.T. Maruszewski, A. Drzewiecki, R. Starosta, L. Restuccia, Thermoelastic damping in an auxetic rectangular plate with thermal relaxation: forced vibrations, Journal of Mechanics of Materials and Structures., 8,(8-10), 403-413 (2013).

[7] J.B. Alblas, A note on the theory of thermoelastic damping, Journal of Thermal Stresses, 4, (3-4), 333-355 (1981).

[8] C. Zener, Internal friction in solids, Physical Review, 52, (3), 230-235 (1937).

[9] J.B. Alblas, On the general theory of thermoelastic friction, Applied Scientific Research A,10, (1), 349-362 (1961).

[10] B. Maruszewski, Nonlinear thermoelastic damping in circular plate, Zeitschrift für Angewandte Mathematik und Mechanik, 72, (4), T75-T78 (1992).

[11] J. Ignaczak, M. Ostoja-Starzewski, Thermoelasticity with finite wave speeds, Oxford University Press, New York 2010.

[12] M. Chester, Second sound in solids, Physical Rev, 131, (5), 2013 (1963).

[13] B. Maruszewski, Evolution equations of thermodiffusion in paramagnets, International Journal of Engineering Science, 26, (11), 1217-1230 (1988).

[14] D. Jou, J. Casas-Vazquez, G. Lebon, Extended irreversible thermodynamics, Reports on Progress in Physics, 51, (8), 1105 (1988).

[15] R. Lakes, Foam structures with a negative Poisson's ratio, Science, 235(4792), 1038-1040 (1987).

[16] K.W. Wojciechowski, Two-dimensional isotropic system with negative Poisson's ratio, Physics Letters A, 137,(1-2), 60-64 (1989).

[17] V.V. Novikov, K.W. Wojciechowski, Negative Poisson coefficient of fractal structures, Physics of the Solid State, 41, 1970-1975 (1999). 
[18] A.A. Pozniak, H. Kamiński, P.Kędziora, B. Maruszewski, T. Stręk, K.W. Wojciechowski, Anomalous deformation of constrained auxetic square, Reviews on Advanced Materials Science, 23,(2), 169-174 (2010).

[19] P. Kolat, B. Maruszewski, K.W. Wojciechowski, Solitary waves in auxetic plates, Journal of Non-Crystalline Solids, 356, 2001-2009 (2010).

[20] P. Kolat, B. Maruszewski, K.V. Tretiakov, K.W. Wojciechowski, Solitary waves in auxetic rods, Physica Status
Solidi (b), 248, (1), 148-157 (2011).

[21] L.D. Landau, E.M. Lifshitz, Theory of elasticity, Pergamon Press 1970.

[22] A. Alderson, K.L. Alderson, K.E. Evans, J.N. Grima, M.R. Wiliams, P.J. Davies, Modelling the deformation mechanisms, structure-property relationships and applications of auxetic nanomaterials, Physica Status Solidi (b), 242, (3) , 499-508 (2005).

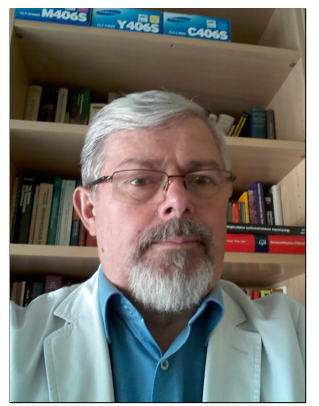

Bogdan T. Maruszewski graduated in physics (1970) from the Adam Mickiewicz University in Poznan, Poland (Ph.D. 1979, Dr. Habil. 1987, Professor 1993) and passed all his scientific carrier steps at Poznan University of Technology. He has acted as deputy head and head of the Institute of Applied Mechanics as well as deputy rector of Poznan University of Technology. His research concerns thermomechanics of continua, coupled fields interactions in solids and thermodynamical modelling in materials of classical and nonclassical properties. He has been member and president of many international and Polish scientific societies and organizations. Author of more than 150 papers in major professional journals being cited ca. 300 times. Frequent visitor in major research centers in Europe, China, USA and Australia. Laureate of the Polonia Restituta Knight's Cross and several other awards for his research and teaching achievements.
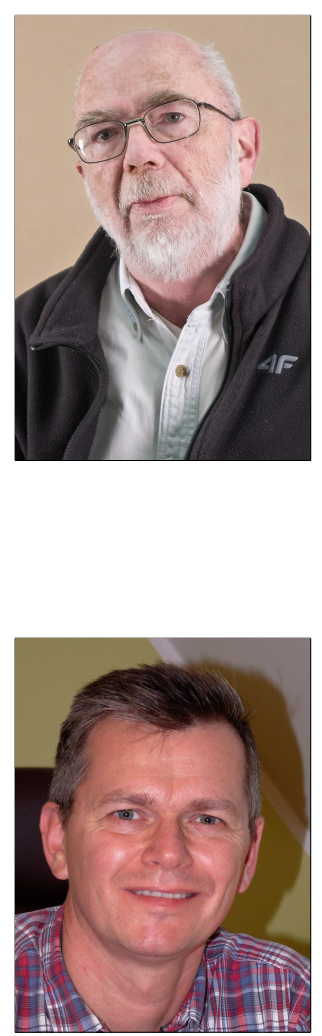

Andrzej Drzewiecki graduated in Physics (1970) at Poznan University (UAM). He attained his Ph.D. at Poznan University of Technology (1979). Currently he is a retired employee of the Institute of Applied Mechanics of Poznan University of Technology. His research interests focus on coupled field theory, piezoelectricity and recently on some dynamic properties of auxetic materials.

Roman Starosta received the $\mathrm{PhD}$ in mechanics in 2000 with a thesis concerning dynamics of an offshore structure under water wave loading. Since 2012 he has been working as Associate Professor at the Faculty of Mechanical Engineering and Management of Poznan University of Technology (PUT), Poland. His research interests include computational nonlinear dynamics, asymptotic methods, and computer programming. He is president of the Poznan division of Polish Society of Theoretical and Applied Mechanics and head of the Division of Technical Mechanics at PUT. He is author and co-author of over 80 papers in major professional journals and conference proceedings. He cooperates with the Department of Automation, Biomechanics and Mechatronics of Lodz University of Technology. 